\title{
Moebius Function on the Lattice of Dense Subgraphs
}

\author{
R. E. Nettleton and M. S. Green
}

\author{
(August 3, 1959)
}

\begin{abstract}
The Moebius function $f_{k}$ on the lattice of $k$-dense subgraphs of a connected graph, defined in a previous paper, is calculated for graphs $G$ containing isthmuses and articulators. $f_{1}$ evaluated for the null graph $\phi$ is shown to vanish if $G$ contains an isthmus, while for any integer $q$ there exist graphs containing articulators for which $f_{1}(\phi)=q$. The "lattice of path sets" joining a pair of points and the lattice of graphs "associated with $G$ and a subgraph $G^{\prime}$ " are defined and the Moebius functions on these lattices are shown in certain cases to be related to $f_{1}$.
\end{abstract}

The concepts " $k$-dense subgraph," "isthmus," and "articulator" were defined in a previous paper [1], where it was shown that the $k$-dense subgraphs of a given connected graph $G$, together with the null graph $\phi$, form a lattice. The Moebius function $f_{k}$ on this lattice will be defined in the present paper, and $f_{k}(\phi)$ and, in particular, $f_{1}(\phi)$ will be evaluated for various types of graphs. It is found that $f_{1}(\phi)=0$ if $G$ contains an isthmus, and $\left|f_{1}(\phi)\right| \leq 1$ unless $G$ contains an articulator. However, for every integer $q$, there exists a graph containing an articulator for which $f_{1}(\phi)=q$. A second lattice is formed by the sets of paths joining a given pair of points and containing among them all the points of $G$, and when $G$ contains an articulator in which there are just two points, $f_{1}(\phi)$ can be expressed in terms of the Moebius function defined on the second lattice. The graphs "associated with a graph $G$ and a subgraph $G^{\prime \prime}$ are defined and shown to form a lattice. A relation is found between the Moebius function on this lattice and the functions $f_{1}(\phi)$ defined for the individual lattice elements.

\section{Definitions}

A linear, undirected graph $G$ is a set of elements, called points, together with a set of ordered pairs of these elements which define a symmetric nonreflexive, binary relation. For any given graph $G$, the number of points will be assumed finite and unless otherwise specified this number will be denoted by the symbol $n$. A subset of the set of points in $\dot{G}$, together with all the ordered pairs which contain only points in the subset, is called a subgraph. The union, intersection, or difference of two subgraphs is the subgraph determined respectively by the union, intersection or difference of the sets of points in the two subgraphs. If $G^{\prime}$ is a subgraph of $G$, we say $G^{\prime}$ is contained in $G$ and write $G^{\prime} \subset G$. 'Two points appearing in the same ordered pair in $G$ are said to

\footnotetext{
1 Figures in brackets indicate the literature references at the end of this paper.
}

be neighbors. For any given pair of points, $p$ and $q$, a sequence of distinct points $\left\{p_{1}, \ldots, p_{r}\right\}$, with the property that $p_{1}=p, p_{r}=q$, and $p_{i}$ and $p_{i+1}$ are neighbors for $1 \leq i<r$, is called a path joining $p$ and $q$, in which $p_{1}$ is the initial point and $p_{\tau}$ the terminal point. A path containing $r$ points will be called an $r$-cycle if its initial and terminal points are neighbors and no point of the path is a neighbor of more than two other points of the path. If the sequence of points of a path $P$ contains a proper subsequence which is also a path, the subsequence is called a subpath contained in $P$. Two points joined by a path are said to be connected. A graph is connected if it has only one point, or more than one point and each pair of points connected. If every pair of points are neighbors, $G$ is said to be completely connected. A graph which not connected is disconnected. The null graph $\phi$ is disconnected. Unless otherwise specified, the symbol $G$ will denote a connected graph.

If $\mathrm{G}^{\prime}$ is a subgraph and $G-G^{\prime}$ is not connected, $G^{\prime}$ is said to disconnect $G$. If $G^{\prime}$ disconnects $G$ and $G-G^{\prime}$ contains, and is contained in, a set of connected graphs, the union of any pair of which is not connected, this set is called the partition of $G-G^{\prime}$. If $G^{\prime}$ contains precisely $m$ points, the partition of $G-G^{\prime}$ consists of at least $k+1$ connected graphs, and $G^{\prime}$ contains no proper subgraph which disconnects $G$, then $G^{\prime}$ is called an $[m, k]$-isthmus if it is completely connected and an $[m, k]$-articulator if it is not completely connected. An $[m, 1]$-articulator or $[m, 1]$ isthmus will be called an articulator or isthmus respectively when the number of points is not relevant.

For any subgraph $G^{\prime}, G\left(G^{\prime}\right)$ will denote the subgraph determined by the set of all points in $G-G^{\prime}$ which have neighbors in $G^{\prime}$. If $G^{\prime}$ is a single point $p$, we shall denote this set by $G(p)$. A connected subgraph $G^{\prime}$ is said to be $k$-dense provided there are at most $k-1$ points in $G-G^{\prime}$ which are not points of $G\left(G^{\prime}\right)$. A $k$-dense subgraph which contains no $k$ dense proper subgraph is $D_{k}$-minimal. A $k$-dense 
proper subgraph of $G$, which is contained in no other $k$-dense subgraph except $G$ itself, is called $D_{k^{-}}$ maximal. The symbol $\Gamma_{k}$ will denote the union of all the $D_{k}$-minimal subgraphs of $G$.

Let $S_{k}$ denote the set of all the $k$-dense subgraphs of $G$ which contain at least $k$ points, together with the null graph. It has been shown in a previous paper [1, theorem 2.2] that the set $S_{k}$ form a lattice under the relation of set inclusion, in which the l.u.b. of two subgraphs is their union; and the g.l.b. of two subgraphs is their intersection, if it is in $S_{k}$, and otherwise is either $\phi$ or a graph in the partition of the intersection, if the latter is not connected. The lattice formed by the graphs in $S_{k}$ will be called the lattice of $k$-dense subgraphs. For any finite lattice composed of elements partially ordered by the relation $\geqslant$ there is a greatest element $I$ such that $I \geqslant x$ for every element $x[2$, ch. II]. The Moebius function on the lattice is a relation which associates with every lattice element $x$ a unique integral number $M(x)$, defined by

$$
\begin{aligned}
& M(I)=1 \\
& M(x)=-\sum_{y>x} M(y)
\end{aligned}
$$

For any lattice element $x, M(x)$ given by this definition is equal to the number given for the same element by the usual definition of the Moebius function $[2$, ch. I] on the dual lattice obtained by replacing $\geqslant$ by $\leq$. On the lattice of subgraphs in $S_{k}$ for which $G$ is the greatest element and $\geqslant$ is understood to mean $\supseteq$, the Moebius function will be denoted by $f_{k}$ and the number which it associates with a given subgraph $G^{\prime}$ by $f_{k}\left(G^{\prime}\right)$. A second function, defined on the entire set of non-null subgraphs of $G$ is the size $\sigma$. For any subgraph $G^{\prime} \neq \phi, \sigma\left(G^{\prime}\right)$ is the number of points in $\dot{G}^{\prime}$.

\section{Completely Connected Graphs and Graphs Containing Isthmuses}

The Moebius function on the lattice of $k$-dense subgraphs will be shown to depend on the size of $G$ and on the way in which the points are connected. First, we shall prove some preliminary combinatorial theorems.

Lemma 2.1. If $G_{1}$ is a subgraph containing a proper 1-dense subgraph $G_{2}$ and $S$ is the set of all subgraphs of $G_{1}$ which are 1-dense and contain $G_{2}$, then ${ }^{2}$

$$
\sum_{G^{\prime} \in S}(-)^{\sigma\left(G^{\prime}\right)}=0
$$

It has been proved [3, lemma 2.1] that a subgraph which contains a 1-dense subgraph is 1-dense. Therefore a subgraph in $S$ is formed by taking $G_{2}$ alone or the union of $G_{2}$ and the subgraph determined by any set of points from $G_{1}-G_{2}$. If $\sigma\left(G_{1}\right)-\sigma\left(G_{2}\right)=m$,

2 The notation $\Sigma_{A \in B}$ will denote the sum over all subgraphs $A$ belonging to the set $B$ of subgraphs, while $\Sigma_{C \subset A \in B}$ will denote the sum over all subgraphs $A$ which belong to $B$ and which contain the subgraph $C$, and $\Sigma_{C \supset} \in B$ the sum over all $A$ in $B$ which are contained in $C$ the number of subgraphs in $G_{1}-G_{2}$ which have $k$ points is $\left(\begin{array}{c}m \\ k\end{array}\right)$ and so

$\sum_{G^{\prime} \in S}(-)^{\sigma\left(G^{\prime}\right)}=(-)^{\sigma\left(G_{2}\right)} \sum_{k=0}^{m}\left(\begin{array}{c}m \\ k\end{array}\right)(-)^{k}=(-)^{\sigma\left(G_{2}\right)}(1-1)^{m}=0$.

A sequence of subgraphs $\left\{\mathrm{H}_{1}, \ldots, H_{q}\right\}$, for any positive integer $q$, will be called an $H$-sequence if, and only if, it has the following properties: (1) $H_{1}$ is connected; (2) for every i> $1, \quad H_{i} \subset \mathrm{G}\left(H_{i-1}\right)$ $-\sum_{i=1}^{i-2} G\left(H_{i}\right)-H_{1}$. If $H_{1}=G^{\prime}$, for a given connected subgraph $G^{\prime}$, the $H$-sequence is said to be based on $G^{\prime}$.

Lemma 2.2. If $\left\{H_{1}, \ldots, H_{q}\right\}$ is an $H$-sequence, then:

1. For any $r \geq 1, \sum_{i=1}^{r} H_{i}$ is connected.

2. If $q>1$ and $\sum_{i=1}^{q} H_{i}$ is 1-dense, then either there exists a non-null subgraph $H_{q+1}$ such that $\left\{H_{1}, \ldots, H_{q}, H_{q+1}\right\}$ is an $H$-sequence, or $\sum_{i=1}^{q-1} H_{i}$ is 1-dense.

To prove part 1 , suppose $p_{k}$ is a point in $H_{k}$ for $k \leq r$. Then $p_{k}$ has a neighbor $p_{k-1}$ in $H_{k-1}$, and given a point $p_{i}$ in $H_{i}$ for $i \leq k-1, p_{i}$ has a neighbor $p_{i-1}$ in $H_{i-1}$. Thus there exists a path $p=\left\{p_{k}\right.$, .

$\left.p_{1}\right\}$ joining $p_{k}$ in $H_{k}$ to a point $p_{1}$ in $H_{1}$ and which contains no other point in $H_{1}$. Similarly, if $p^{\prime}$ is any point in $\sum_{i=1}^{r} H_{i}$ distinct from $p_{k}, p^{\prime}$ is in some $H_{a}$ for $a \leq r$, and there exists a path $P^{\prime}$ joining $p^{\prime}$ to a point $\bar{p}_{1}{ }^{\prime}$ in $H_{1}$ and containing no other point in $H_{1}$. Since $H_{1}$ is connected, there is a path $P_{1}$ in $H_{3}$ joining $p_{1}$ and $p_{1}{ }^{\prime}$. If these three paths are all disjoint, i.e., no two have any point in common except for the distinct points $p_{1}$ and $p_{1}{ }^{\prime}$, they form, taken together, a path $\left\{p_{k}, \ldots, p_{1}, \ldots, p_{1}{ }^{\prime}, \ldots, p^{\prime}\right\}$ joining $p_{k}$ and $p^{\prime}$. If these paths are not disjoint except for distinct $p_{1}$ and $p_{1}^{\prime}$ there exists a point $p_{b}$ in $P \cap H_{b}$ which is also a point of $P^{\prime}$. If $b$ is the greatest integer for which this is the case, then the segments $\left\{p_{k}, \ldots, p_{b}\right\}$ and $\left\{p^{\prime}, \ldots, p_{b}\right\}$, of $P$ and $p^{\prime}$ respectively, when taken together, form a path $\left\{p_{k}, \ldots, p^{\prime}\right\}$ joining $p_{k}$ and $p^{\prime}$.

To prove part 2 , suppose that there is no non-null $H_{q+1}$ such that $\left\{H_{1}, \ldots, H_{q}, H_{q+1}\right\}$ is an $H$-sequence. Then every point in $G\left(H_{q}\right)$ has a neighbor in one of the $H_{i}$ for $1 \leq i<q$. Furthermore every point in $H_{q}$ has a neighbor in $H_{q-1}$. From part 1 , we know that $\sum_{i=1}^{q-1} H_{i}$ is connected and since it contains a neighbor of every point in $H_{q}$ and of every point which has a neighbor in $\sum_{i=1}^{q} H_{i}$, it must also be 1-dense.

Lemma 2.3. If $G^{\prime \prime}$ is a connected subgraph and $G^{\prime}$ is a 1-dense subgraph containing $G^{\prime \prime}$, then there is a unique $H$-sequence based on $G^{\prime \prime}$ such that $G^{\prime}$ is equal to the union of all the subgraphs in the H-sequence.

Define $H_{1} \equiv G^{\prime \prime}$ and for $i>1$ let $H_{i} \equiv G^{\prime} \cap G\left(H_{i-1}\right)$ $-\sum_{j=1}^{i-2} G\left(H_{j}\right)-H_{1}$. Suppose that for some $r$, there are points of $G^{\prime}$ not in $\sum_{i<r} H_{i}$. At least one of these points, $p$ say, must have a neighbor in some $H_{j}$ for some $j \leq r$ since otherwise $G^{\prime}$ would not be connected. If $\bar{j}<r, p$ must be in $H_{i}$ for $i \leq r$. This is contrary to hypothesis, and so $p$ is in $H_{r+1}$. Thus since $G^{\prime}$ has only a finite number of points, there must be a positive integer $q$ such that $G^{\prime} \subset \sum_{i=1}^{q} H_{i}$, 
and it follows that $G^{\prime}=\sum_{i=1}^{q} H_{i}$, since the $H_{i}$ contain only points of $G^{\prime}$. Furthermore the sequence $\left\{H_{1}, \ldots, H_{q}\right\}$, by its construction, is an $H$-sequence. Suppose that there is a second $H$-sequence $\left\{H_{1}^{\prime}, \ldots, H_{m}^{\prime}\right\}$ based on $G^{\prime \prime}$ such that $G^{\prime}=\sum_{i=1}^{m} H_{i}{ }^{\prime}$, $H_{i}=H_{i}^{\prime}$ for $i<j$ and $H_{j} \neq H_{j}^{\prime}$. It then follows that $H_{j}^{\prime}$ must be unequal to $G^{\prime} \cap G\left(H_{j-1}\right)-\sum_{i=1}^{j-2} G$ $\left(H_{i}\right)-H_{1} \equiv H_{j}$. We have $H_{j}^{\prime} \subset H_{j}$, and therefore there are points in $H_{3}-H_{j}^{\prime}$ which are in graphs $H_{k}^{\prime}$ for $k>j$. Since these points have neighbors in $H_{j-1}^{\prime}$, this result is contrary to the definition of an $H$-sequence and thus $H_{j}=H_{j}^{\prime}$. Since $H_{1}=H_{1}^{\prime}=G^{\prime \prime}$, it follows by induction that the two $H$-sequences are identical.

An $H$-sequence based on a connected subgraph $G^{\prime \prime}$ with the property that the union of all its graphs is 1 -dense will be called a $D$-minimal $H$-sequence if it contains no proper subsequence which is also an $H$-sequence based on $G^{\prime \prime}$ with this property. By 2 . 3 , for a given connected subgraph $G^{\prime \prime}$ in $G$, to every 1-dense subgraph $G^{\prime}$ containing $G^{\prime \prime}$ there corresponds a unique $H$-sequence based on $G^{\prime \prime}$ such that $G^{\prime}$ is the union of the graphs in the $H$-sequence. This $H$-sequence contains a subsequence which is a $D$ minimal $H$-sequence based on $\mathrm{G}^{\prime \prime}$ and which is also unique. We can see this by observing that given two $H$-sequences $\left\{H_{i}{ }^{\prime}\right\}$ and $\left\{H_{i}^{\prime \prime}\right\}$ which are subsequences of an $H$-sequence $\left\{H_{i}\right\}$, if for some $r$, $H_{j}^{\prime}=H_{j}^{\prime \prime}=H_{j}$ for all $j \leq r$ and $H_{r+1}^{\prime} \neq H_{r+1}^{\prime \prime}$, then either $H_{r+1}^{\prime}$ or $H_{r+1}^{\prime \prime}$ is $\neq H_{r+1}$ and one of these must be equal to $H_{j}$ for $j>r+1$, which is impossible by definition of an $H$-sequence, Thus $\left\{H_{i}^{\prime}\right\}$ and $\left\{H_{i}^{\prime \prime}\right\}$ must be identical if they are both $D$-minimal. Accordingly the $H$-sequences based on $G^{\prime \prime}$ and corresponding to 1 -dense subgraphs containing $G^{\prime \prime}$ may be divided into families such that all the $H$-sequences in each family contain a particular $D$-minimal $H$ sequence based on $G^{\prime \prime}$, and then the $H$-sequence corresponding to a particular 1-dense subgraph containing $G^{\prime \prime}$ will belong to one, and only one, family. Let $S$ be the set of all 1-dense subgraphs for which the corresponding $H$-sequences belong to a particular family; let $\left\{H_{i}, \ldots, H_{q}\right\}$ be the $D$-minimal $H$-sequence contained in every $H$-sequence of the family; and let $G_{2}=\Sigma_{i=1}^{q} H_{i}$. Then every $H$-sequence in the family may be denoted by $\left\{H_{1}, \ldots, H_{q}, H_{q+1}\right\}$ where $H_{q+1}$ is the graph determined by any set of points, empty or nonempty, in $G\left(H_{q}\right)-\sum_{i=1}^{q-1} G\left(H_{i}\right)-$ $H_{1} \equiv G_{1}$. There can be no $H_{k}$ for $k>q+1$ in any $H$-sequence of the family, since otherwise $H_{k}$ would have a neighbor in some $H_{i}$ for $i \leq q$, which is impossible. By part 2 of 2.2 , we know that $G_{1}$ is nonnull if $q>1$. If $q=1, G_{1}$ is non-null if $G^{\prime \prime}=H_{1}$ is a proper subgraph. Assume, therefore that $G^{\prime \prime} \neq G$, so that $G_{1} \neq \phi$. The set $S$ is the set of all 1 -dense subgraphs in $G_{1} \cup G_{2}$ which contain $G_{2}$. By 2.1, $\sum_{G^{\prime} \epsilon S}(-)^{\sigma\left(G^{\prime}\right)}=0$. and this must be true of every family of $H$-sequences based on $G^{\prime \prime}$. Thus if $S_{a}$ is the set of all 1 -dense subgraphs which contain $G^{\prime \prime}$, $\sum_{G^{\prime} \in S a}(-)^{\sigma\left(G^{\prime}\right)}=0$. This establishes the theorem:

THEOREM 2.4. If $G^{\prime \prime}$ is a proper connected subgraph in $G$ and $S_{a}$ is the set of all 1-dense subgraphs which contain $G^{\prime \prime}$, then $\sum_{G^{\prime} \epsilon S_{a}}(-)^{\sigma\left(G^{\prime}\right)}=0$.
The preliminary theorems and lemmas are now established which will make possible the determination of the value of $f_{k}$ corresponding to any particular lattice point. Since the Moebius function is uniquely defined, any function must be equal to $f_{k}$ if it satisfies the equations which define $f_{k}$ recursively on the lattice of $k$-dense subgraphs. Consider in particular the function $g_{k}$ whose domain is the set of subgraphs in $S_{k}$, defined by

$$
g_{k}\left(G^{\prime}\right)=(-)^{n+\sigma\left(G^{\prime}\right)}
$$

for $\phi \neq G^{\prime} \epsilon S_{1} \cap S_{k}$,

$$
g_{k}\left(G^{\prime}\right)=0
$$

if $G^{\prime}$ is in $S_{k}-\phi$ but not 1 -dense, and

$$
g_{k}(\phi)=(-)^{n+1} \sum_{\phi \neq \neq G^{\prime} \in S_{1} \cap S_{k}}(-)^{\sigma\left(G^{\prime}\right)} .
$$

Since every subgraph which contains a 1-dense subgraph is 1-dense and thus $k$-dense, the set $S$ of all subgraphs in $S_{k}$ which contain a given 1-dense subgraph $G^{\prime \prime}$ in $S_{k}$ is the set of all subgraphs which contain $G^{\prime \prime}$. Thus

$$
\begin{array}{r}
-\sum_{G^{\prime \prime}<G^{\prime} \in S_{k}-G^{\prime \prime}} g_{k}\left(G^{\prime}\right)=(-)^{n+1}\left[\sum_{G^{\prime} \in S}(-)^{\sigma\left(G^{\prime}\right)}-(-)^{\sigma\left(G^{\prime \prime}\right)}\right] \\
=(-)^{n+\sigma\left(G^{\prime \prime}\right)}=g_{k}\left(G^{\prime \prime}\right) .
\end{array}
$$

which follows by 2.1 if we set $G_{1}=G$ and $G_{2}=G^{\prime \prime}$. If $G^{\prime \prime} \epsilon S_{k}-S_{1}$, the set of all subgraphs which properly contain $G^{\prime \prime}$ and for which $g_{k}$ does not vanish is identical with the set of $S$ of all 1-dense subgraphs containing $G^{\prime \prime}$. Since $G^{\prime \prime}$ is not 1 -dense, $G^{\prime \prime} \neq G$, and since $G^{\prime \prime}$ is $k$-dense, it is connected. Therefore, by 2.4

$$
-\sum_{G^{\prime \prime} \subset G^{\prime} \in S_{k}-G^{\prime \prime}} g_{k}\left(G^{\prime}\right)=(-)^{n+1} \sum_{G^{\prime} \in S}(-)^{\sigma\left(G^{\prime}\right)}=0=g_{k}\left(G^{\prime \prime}\right) .
$$

Thus it is established that $g_{k}\left(G^{\prime}\right)$ satisfies the recurrence relations which define $f_{k}$ for all $G^{\prime} \neq \phi$ in $S_{k}$, and so $g_{k}\left(G^{\prime}\right)=f_{k}\left(G^{\prime}\right)$ for $G^{\prime} \neq \phi$. It then follows from its definition that $g_{k}(\phi)=f_{k}(\phi)$. Accordingly, we have proved

Theorem 2.5. On any lattice of $k$-dense subgraphs, $g_{k}=f_{k}$.

Since for any non-null $G^{\prime}$ in $S_{k}, f_{k}\left(G^{\prime}\right)$ depends only on $\sigma\left(G^{\prime}\right)$ and on whether or not $G^{\prime} \epsilon S_{1}$, attention will be given to $f_{k}(\phi)$, and in particular $f_{1}(\phi)$, for which the value depends on whether $G$ is completely connected, or contains an articulator or isthmus, and on other properties of $G$.

Theorem 2.6. If $G$ is completely connected, $f_{1}(\phi)=(-)^{n}$.

If $G$ is completely connected, every point is a neighbor of all other points and is therefore 1-dense, as, in fact, is every non-null subgraph. Thus, for every $k \geq 1, G$ contains $\left(\begin{array}{l}n \\ k\end{array}\right)$ 1-dense subgraphs hav- 
ing $k$ points each, so that by 2.5 ,

$$
f_{1}(\phi)=(-)^{n+1} \sum_{k=1}^{n}\left(\begin{array}{l}
n \\
k
\end{array}\right)(-)^{k}=(-)^{n},
$$

a result which follows from the binomial expansion of $(1-1)^{n}$.

Lemma 2.7. If $S$ is a set of subgraphs of $G, F_{i}$ for $i=1,2, \ldots ., n$ the set of all families of subgraphs in $S$ such that each family contains precisely $i$ subgraphs, for any particular family $C, B(C)$ is the union of all the subgraphs in $C$, and $S^{\prime}$ is the set of all subgraphs of $G$ which belong to a given set $R$ and which contain one of the subgraphs in $S$, then

$$
\sum_{G^{\prime} \in S^{\prime}}(-)^{\sigma\left(G^{\prime}\right)}=-\sum_{i=1}^{n} \sum_{C \in F_{i}} \sum_{B(C) \subset G^{\prime \prime} \epsilon R}(-)^{\sigma\left(G^{\prime \prime}\right)+i} .
$$

Consider a particular subgraph $G^{\prime} \epsilon S^{\prime}$ and suppose that $G^{\prime}$ contains precisely $q$ subgraphs from $S$ which form a family $F_{q}$. The subgraph $G^{\prime}$ will occur once in the triple sum in (A) for every subfamily of the family $F_{q}$, and since $F_{q}$ contains $\left(\begin{array}{l}q \\ k\end{array}\right)$ subfamilies of precisely $k$ graphs each, the total contribution to the triple sum in (A) of the summands corresponding to $G^{\prime}$ is

$$
-\sum_{k=1}^{q}\left(\begin{array}{l}
q \\
k
\end{array}\right)(-)^{k+\sigma\left(G^{\prime}\right)}=(-)^{\sigma\left(G^{\prime}\right)}
$$

Since this result holds for every subgraph $G^{\prime}$ in $S^{\prime}$, the right and left members of (A) are equal.

Theorem 2.8. If $G$ contains at least $k$ disjoint subgraphs, each of which disconnects $G$ and at least one of which is an isthmus, $f_{k}(\phi)=0$.

If $G_{1}, \ldots . ., G_{k}$ are $k$ disjoint subgraphs each of which disconnects $G$, then every 1-dense subgraph contains at least $k$ points, one from each $G_{i}$ $(i=1, \ldots, k)$. Otherwise for some $j,(1 \leq j \leq k)$, $G-G_{j}$ would be 1-dense and thus connected. Thus $S_{1}=S_{1} \cap S_{k}$ and $f_{k}(\phi)=g_{k}(\phi)=g_{1}(\phi)$ by 2.5. Suppose one of the $G_{i}$, $G_{1}$ say, is completely connected, so that every subgraph of $G_{1}$ is a connected proper subgraph of $G$. If $S$ is the set of non-null subgraphs of $G_{1}$ and $S^{\prime}$ the set of 1-dense subgraphs containing a graph from the set $S$, we have $S^{\prime}=S_{1}$, and thus

$$
g_{1}(\phi)=(-)^{n+1} \sum_{G \epsilon S^{\prime}}(-)^{\sigma\left(G^{\prime}\right)} .
$$

If $C$ is any subfamily of $S$ and $B(C)$ the union of the graphs in $C, B(C)$ is connected because $G_{1}$ is completely connected, and thus by 2.4 ,

$$
\sum_{B(C) \subset G^{\prime \prime} \epsilon S_{1}}(-)^{\sigma\left(G^{\prime \prime}\right)}=0
$$

Since this is true of every family $C$, we find from 2.7 that $g_{1}(\phi)=0$.
Let us now suppose that $S$ denotes the set of minimal subgraphs which belong to $S_{1} \cap S_{k}$, i.e., the subgraphs with this property which have no proper subgraphs belonging to $S_{1} \cap S_{k}$, while $R$ is the set of 1 -dense subgraphs. Since every 1-dense subgraph having at least $k$ points contains a minimal subgraph with this property, 2.5 implies if $S^{\prime}$ is the set of all subgraphs in $R$ which contain one of the subgraphs in $S$, then

$$
f_{k}(\phi)=(-)^{n+1} \sum_{G^{\prime} \in S^{\prime}}(-)^{\sigma\left(G^{\prime}\right)} .
$$

If $C$ is any family of subgraphs in $S$ and $B(C)$ is the union of the graphs in $C$, then $B(C)$ is connected, since the union of two 1-dense subgraphs is 1-dense [2, lemma 2.1] and therefore connected. If $G$ is not equal to the union of all the subgraphs in $S$, $B\left(C^{\gamma}\right)$ is a proper connected subgraph. Then by 2.4 ,

$$
\sum_{B(C) \in G^{\prime \prime} \epsilon R}(-)^{\sigma\left(G^{\prime}\right)}=0
$$

Since this holds for every family C, 2.7 implies

$$
\sum_{G^{\prime} \epsilon S^{\prime}}(-)^{\sigma\left(G^{\prime \prime}\right)}=0
$$

so that $f_{k}$ vanishes. Thus we have proved

Theorem 2.9. If $G$ is not equal to the union of all the minimal subgraphs with the property that they are 1 -dense and contain at leasi $k$ points, then $f_{k}(\phi)=0$.

From 2.9 is is seen that $f_{1}(\phi)=0$ unless $G$ is equal to the union of the $D_{1}$-minimal subgraphs. If $G=\Gamma_{1}$ and that $D_{1}$-minimal subgraphs are mutually disjoint, it has been proved elsewhere [3, theorem 2.6] that $G$ is completely connected, so that, by 2.6 , $f_{1}(\phi)=(-)^{n}$.

Thus we have proved

Theorem 2.10. If the $D_{1}$-minimal subgraphs are mutually disjoint, $\left|f_{1}(\phi)\right| \leq 1$.

It has been proved [3, theorem 2.4] that if $n>1, G$ contains at least two $D_{1}$-maximal subgraphs. Suppose $n>1$ and there is a $D_{1}$-minimal subgraph $G^{\prime}$ which is contained in every $D_{1}$-maximal sugraph, and $G^{\prime \prime}$ is $D_{1}$-minimal, $G^{\prime \prime} \neq G^{\prime}$. Then there is a point $p$ in $G^{\prime}-G^{\prime \prime}$, and $G^{\prime \prime}+(G-p)$ is $D_{1}$-maximal. This impossible, since this $D_{1}$-maximal subgraph does not contain $G^{\prime}$. Thus $\Gamma_{1}=G^{\prime}$, and $\Gamma_{1}$ is a proper subgraph, so that by $2.9, f_{1}(\phi)=0$. This result is Hall's theorem [4] for the special case of the lattice of 1-dense subgraphs. It may be stated in the form:

Theorem 2.11. On the lattice of 1-dense subgraphs of $G$, if $n>1$ and $\phi$ is not the g.l.b. of any set of $D_{1}$-maximal subgraphs, then $f_{1}(\phi)=0$.

It has been previously proved [3] that either $G$ is completely connected, in which case $\left|f_{1}(\phi)\right|=1$ by 2.6 , or $G$ contains a disconnecting subgraph which in turn must contain an articulator or an isthmus. If $G$ contains an isthmus, $f_{1}(\phi)=0$ by 2.7 , and thus we have proved

Theorem 2.12. $\left|f_{1}(\phi)\right| \leq 1$ unless $G$ contains an articulator. 


\section{Graphs Containing Articulators}

A simple example of a graph containing an articulator is an $r$-cycle for $r>3$. Since each point is connected to only two other points, the two neighbors of any given point in the $r$-cycle constitute a disconnecting subgraph $G^{\prime}$, which is not connected, each point of which is connected to both graphs in the partition of $G-G^{\prime}$, and which is therefore a $[2,1]$-articulator.

Theorem 3.1. If $G$ contains an $n$-cycle, then $f_{k}(\phi)=-1$ for $k \neq n-1$, and $f_{n-1}(\phi)=n-1$.

If $G$ contains an $n$-cycle, every point is one of a sequence $\left\{p_{1}, \ldots, p_{n}\right\}$ such that each point is a neighbor only of those which immediately precede and immediately follow in the sequence, except for $p_{1}$ and $p_{n}$ which are neighbors. We have from 2.5, $f_{n}(\phi)=(-)^{n+1} \cdot(-)^{n}=-1$. For any $i, G-p_{i}$ is 1 -dense since $P \equiv\left\{p_{i+1}, \ldots, p_{n}, p_{1}, \ldots, p_{i-1}\right\}$ is a path connecting $p_{i+1}$ and $p_{i-1}, p_{i-1}$ being taken to mean $p_{n}$ if $i=1$, which are neighbors of $p_{i}$, and any two other points in $G-p_{i}$ are joined by a subpath contained in $P$. Similarly for any $i, G-p_{i}-p_{i-1}$ is connected since there exists a path $\left\{p_{i+1}, \ldots, p_{i-2}\right\}$ containing all the other points. On the other hand, if $p_{a}$ and $p_{b}$ are not neighbors and $a<b$, there exists $p_{c}$ and $p_{d}$ such that $a<c<b$ and either $d>b$ or $d<a$. It is easily shown by induction that any path joining $p_{c}$ and $p_{d}$ must contain $p_{a}$ or $p_{b}$, and thus $G-p_{a}-p_{b}$ is not connected. Thus for $n \geq 3, S_{1}-\phi$ consists of $G$ plus $n$ subgraphs $G-p_{i}(i=1, \ldots, n)$, and $n$ subgraphs $G-p_{i}-p_{i-1}$, so that by $2.5 \quad f_{1}(\phi)=$ $(-)^{n+1}\left[n(-)^{n-2}+n(-)^{n-1}+(-)^{n}\right]=-1$. Also for $n \geq 2$ we have $f_{n-1}(\phi)=(-)^{n+1}\left[n(-)^{n-1}+(-)^{n}\right]=n-1$.

All graphs considered hitherto have been such that $\left|f_{1}(\phi)\right| \leq 1$. Consideration of graphs containing articulators, however, will show that there exist graphs for which $f_{1}(\phi)$ assumes arbitrarily large positive or negative values. First we must prove

Lemma 3.2 If $G$ contains an articulator $G^{\prime}$ such that each point in $G-G^{\prime}$ is a neighbor of every point of $G^{\prime}$ then

$$
f_{1}(\phi)=(-)^{n+1}\left[1+\sum_{G^{\prime} \supset G_{G^{\prime \prime} \epsilon S_{1}-\phi}}(-)^{\sigma\left(G^{\prime \prime}\right)}\right] .
$$

If $H$ is any subgraph containing a point $p^{\prime}$ in $G^{\prime}$ and a point $p$ in $G-G^{\prime}$, then $p$ and $p^{\prime}$ are neighbors; every point in $G^{\prime}$ is a neighbor of $p$; and every point in $G-G^{\prime}$ is a neighbor of $p^{\prime}$. Thus the subgraph $p+p^{\prime}$ is 1 -dense as is $H$ which contains it. If $\sigma\left(G^{\prime}\right)=m$, there are $\left(\begin{array}{l}m \\ k\end{array}\right)$ subgraphs contained in $G^{\prime}$ having $k$ points each and $\left(\begin{array}{c}n-m \\ r\end{array}\right)$ subgraphs contained in $G-G^{\prime}$ having $r$ points each which can be combined to give $\left(\begin{array}{l}m \\ k\end{array}\right) \cdot\left(\begin{array}{c}n-m \\ r\end{array}\right)$ 1-dense subgraphs, each with $k$ points in $G^{\prime}$ and $r$ points in $G-G^{\prime}$. If $S_{1}^{\prime}$ is the set of all graphs in $S_{1}$ which have points in both $G^{\prime}$ and $G-G^{\prime}$, we have

$$
\sum_{H \in S_{1}^{\prime}}(-)^{\sigma(H)}=\left[\sum_{k=1}^{m}\left(\begin{array}{l}
m \\
k
\end{array}\right)(-)^{k}\right]\left[\sum_{r=1}^{n-m}\left(\begin{array}{c}
n-m \\
r
\end{array}\right)(-)^{r}\right]=1 .
$$

Since there are no 1-dense subgraphs contained in $G-G^{\prime}$, which would otherwise be connected, every graph in $S_{1}-S_{1}^{\prime}$ is contained in $G^{\prime}$. Thus, by 2.5 ,

$$
\begin{aligned}
f_{1}(\phi)=(-)^{n+1}\left[\sum_{H \in S_{1}^{\prime}}(-)^{\sigma(H)}+\sum_{G^{\prime} \supset G^{\prime \prime} \epsilon S_{1}-\phi}(-)^{\sigma\left(G^{\prime \prime}\right)}\right] \\
=(-)^{n+1}\left[1+\sum_{G^{\prime} \supset G^{\prime \prime \prime} \epsilon S_{t}-\phi}(-)^{\sigma\left(G^{\prime \prime}\right)}\right] .
\end{aligned}
$$

Theorem 3.3. If $q$ is any positive integer, there exist graphs $G$ and $G^{\prime}$ for which $f_{1}(\phi)=q$ and $f_{1}(\phi)=-q$ respectively.

Suppose there exists a graph $G_{a}$ for which $f_{1}(\phi)=r$. Consider the graph $G_{b}$ which has the following properties: $G_{b}$ consists of an articulator $G_{b}^{\prime}$ isomorphic with $G_{a}$ plus $s$ points, $p_{1}, \ldots, p_{s}$ at least two of which are not neighbors, and each of which is a neighbor of every point of $G_{b}^{\prime}$. By 3.2, we have for $G_{b}$ the result that

$$
f_{1}(\phi)=(-)^{\sigma\left(G_{a}\right)+s+1}\left[1+{ }_{G_{b}^{\prime} \supset} \sum_{G^{\prime \prime} \epsilon S_{1}-\phi}(-)^{\sigma\left(G^{\prime \prime}\right)}\right] .
$$

Since any subgraph which is 1-dense in $G_{b}^{\prime}$ contains neighbors of $p_{1}, \ldots, p_{s}$ and is therefore 1-dense in $G_{b}$, it follows that

$$
f_{1}^{b}(\phi) \equiv(-)^{\sigma\left(G_{a}\right)+1} \sum_{G^{\prime}{ }^{\prime} \supset G^{\prime \prime \prime} \epsilon S_{1}-\phi}(-)^{\sigma\left(G^{\prime \prime}\right)}
$$

is the function $f_{1}(\phi)$ defined on the lattice of 1dense subgraphs of $G_{b}^{\prime}$. Furthermore, if two graphs are isomorphic, to every 1-dense subgraph of one there corresponds a unique 1-dense subgraph of the other, and thus $f_{1}(\phi)$ for $G_{b}^{\prime}$ is equal to $f_{1}(\phi)$ for $G_{a}$, which we shall denote by $f_{1}^{a}(\phi)$. Thus

$$
f_{1}(\phi)=(-)^{\sigma\left(G_{a}\right)+s+1}+(-)^{s} f_{1}^{a}(\phi) .
$$

If $\sigma\left(G_{a}\right)$ is even, $f_{1}(\phi)=(-)^{s}[r-1]$. In particular we can suppose $G_{a}$ contains an $n$-cycle, $n$ even and $>2$, so that $r=-1$ and let $s$ be odd, so that $\sigma\left(G_{b}\right)$ is odd and $f_{1}(\phi)=2$. If $s$ were even, then $f_{1}(\phi)=-2$. However, we have also proved that if there exists $G_{a}$ such that $\sigma\left(G_{a}\right)$ is odd and $f_{1}^{a}(\phi)=r$, then there exists a graph $G_{a}^{\prime}$ for which $f_{1}(\phi)=(-)^{s}[r+1]$, containing $\sigma\left(G_{a}\right)+s$ points. If $s$ is even, $\sigma\left(G_{a}^{\prime}\right)$ is odd and for $G_{a}^{\prime}, f_{1}(\phi)=r+1$. If $s$ is odd, $f_{1}(\phi)$ for $G_{a}^{\prime}$ is $-[r+1]$. By induction, it follows that for any integer $q$ such that $q=-1$ or $|q| \geq 2$, there exists a graph $G$ for which $f_{1}(\phi)=q$. If $\bar{G}$ is completely connected and $n=2, f_{1}(\phi)=1$ by 2.6 , and thus the theorem is proved for all positive integers $q$. 
TheOREM 3.4. If $p$ and $p^{\prime}$ are two distinct points of $G$, and $S$ is the set of all families of paths joining $p$ and $p^{\prime}$ such that each family contains $n$ points among them, then the families of $S$, together with the null family, $\phi_{S}$, form a lattice under the relation of set inclusion.

If a family $F$ of paths contains all points of $G$, any family containing $F$ is also in $S$, and thus the l.u.b. of two families in $S$ is their union. If the intersection of two families in $S$ is not in $S$, it cannot contain any family of $S$. Thus the g.l.b. of two families is either their intersection or $\phi_{S}$. For any pair of distinct points $p$ and $p^{\prime}$, the lattice formed by the families of paths which join $p$ and $p^{\prime}$, such that the paths of each family contain all points of $G$, will be called a lattice of path sets associated with $G$ and joining $p$ and $p^{\prime}$. For any given lattice formed by a set of $S$ of path sets joining a given pair of points, $\tau(F)$ will denote the number of distinct paths in each lattice element $F \neq \phi_{S}$, two paths being called "distinct" if they differ in at least one point, and $m_{S}$ will denote the greatest positive integer with the property that for some $F, m_{S}=\tau(F)$. The symbol $h_{S}$ will denote the Moebius function on the lattice formed by path sets from $S$.

Theorem 3.5. If $S$ is a set of families of paths, including the null family, which form a lattice of path sets associated with $G$, then for each $F \epsilon S$,

$$
h_{\mathrm{S}}(F)=(-)^{m_{s}+\tau(F)}
$$

for $F \neq \phi_{S}$, and if $m_{S} \geq 1$,

$$
h_{S}\left(\phi_{S}\right)=(-)^{m_{S}+1} \sum_{\phi S \neq F \in S}(-)^{\tau^{(F)}} .
$$

The proof is very similar to that of 2.5 and will be left to the reader.

If $G$ contains a $[2, k]$-articulator consisting of points $p$ and $p^{\prime}$, every 1-dense subgraph must contain either $p$ or $p^{\prime}$ since otherwise $G-p-p^{\prime}$ would be 1-dense and therefore connected. Accordingly if $R$ is the set of 1-dense subgraphs in $G$ and $S^{\prime}$ the set of all subgraphs in $R$ which contain $p, p^{\prime}$, or $p+p^{\prime}$, we have $R=S^{\prime}$, and then 2.5 and 2.7 imply that

$$
\begin{array}{r}
f_{1}(\phi)=(-)^{n+1} \sum_{G^{\prime} \in S^{\prime}}(-)^{\sigma\left(G^{\prime}\right)}=(-)^{n+1} \times\left[\sum_{p \subset G^{\prime \prime} \epsilon R}(-)^{\sigma\left(G^{\prime \prime}\right)}\right. \\
\left.+\sum_{p^{\prime} \subseteq G^{\prime \prime} \in R}(-)^{\sigma\left(G^{\prime \prime}\right)}-\sum_{p+p^{\prime} \in G^{\prime \prime} \in R}(-)^{\sigma\left(G^{\prime \prime}\right)}\right] .
\end{array}
$$

The first two terms in the brackets vanish by 2.4 since $p$ and $p^{\prime}$ are each connected proper subgraphs. Every 1-dense subgraph containing both $p$ and $p^{\prime}$ must contain a path from the set $S$ of all paths joining these two points, and so again employing 2.7, we find that

$$
f_{1}(\phi)=(-)^{n+1} \sum_{i=1}^{n} \sum_{C \in F_{i}} \sum_{B(C) \subset G^{\prime \prime} \epsilon R}(-)^{\sigma\left(G^{\prime \prime}\right)+i},
$$

where $F_{i}$ is the set of all $i$-ples of distinct paths from $S$, and $C, B(C)$, are defined as in the hypothesis of 2.7. For each family $C, B(C)$ is connected since the union of two connected subgraphs having a point in common is connected. Accordingly, by 2.4, $\sum_{B(C) \subset G^{\prime \prime} \in R}(-)^{\sigma\left(G^{\prime \prime}\right)}=0$ unless $B(C)=G$, i.e., unless $C$ is an element of the lattice of path sets joining $p$ and $p^{\prime}$, in which case $\sum_{B(C) \subset G^{\prime \prime} \epsilon R}(-)^{\sigma\left(G^{\prime \prime}\right)+i}=$ $(-)^{n+\tau(C)}$. If $S^{\prime \prime}$ is the set of lattice elements, then $f_{1}(\phi)=-\sum_{\phi s^{\prime \prime} \neq F \in S^{\prime \prime}}(-)^{\tau(F)}$, provided $\phi_{S^{\prime \prime}}$ is not the only element in $S^{\prime \prime}$, and $f_{1}(\phi)=0$ otherwise. This result implies

Theorem 3.6. If $G$ contains a $[2, k]$-articulator and $S$ is the set of elements forming the lattice of path sets associated with $G$ and joining the points of the articulator, then $f_{1}(\phi)=(-)^{m_{s}} h_{S}\left(\phi_{S}\right)$ if $S$ contains a nonempty set of paths, and $f_{1}(\phi)=0$ otherwise.

Theorem 3.6 can be used to show that if $G$ contains a $[2, k]$-articulator consisting of points $p$ and $p^{\prime}$ and $f_{1}(\phi) \neq 0$, then $f_{1}(\phi)$ can be written as a product of factors, one factor for each connected graph in the partition of $G-G^{\prime}$. Any path connecting $p$ and $p^{\prime}$ cannot contain points from more than one graph in the partition of $G-G^{\prime}$, and so any set of paths forming one of the set $S$ of elements of the lattice of path sets joining $p$ and $p^{\prime}$ must contain a subset contained in $G_{i}+p+p^{\prime}$ and containing all the points of $G_{i}$, for each connected graph $G_{i}(i=1, \ldots, w \geq$ $k+1)$ in the partition of $G-G^{\prime}$. Thus if $S_{i}(i=1$, . . . w wenotes the elements of the lattice of path sets associated with $G_{i}+p+p^{\prime}$ and joining $p$ and $p^{\prime}$, we have by 3.5 ,

$$
\begin{array}{r}
h_{S}\left(\phi_{S}\right)=(-)^{m_{S}+1} \prod_{i=1}^{w}\left(\sum_{\phi_{i} \neq G^{\prime \prime} \epsilon S_{i}}(-)^{\tau\left(G^{\prime \prime}\right)}\right)=(-)^{m_{S}+1} \prod_{i=1}^{w} \\
\left\{(-)^{m_{S i}+1} h_{S_{i}}\left(\phi_{S_{i}}\right)\right\} .
\end{array}
$$

Since $m_{S}=\sum_{i} m_{S_{i}}$, we have established

Theorem 3.7. If $G$ contains a $[2, k]$-articulator $G^{\prime}$, $G_{i}$ for $i=1, \ldots, k+1$ are the connected graphs in the partition of $G-G^{\prime}$, and for each $G_{i}, S_{i}$ is the set of elements which form the lattice of path sets associated with $G_{i} \cup G^{\prime}$ and joining the points of the articulator, then $f_{1}(\phi) \neq 0$ implies

$$
f_{1}(\phi)=(-)^{m_{s}+k} \prod_{i=1}^{k+1} h_{S_{i}}\left(\phi_{S_{i}}\right) \cdot
$$

\section{Associated Graphs}

Given a graph $G$ and a subgraph $G^{\prime}$ which is not properly contained in a completely connected subgraph, the set of graphs associated with $G$ and $G^{\prime}$ is defined to be set of all graphs $G^{\prime \prime}$ which have the following properties: (1) A one-one correspondence exists between the points of $G$ and those of $G^{\prime \prime}$ such 
that neighbors in $G$ are mapped into neighbors in $G^{\prime \prime} ;(2) G^{\prime \prime}$ contains a maximal completely connected subgraph, which will be called $K\left[G^{\prime \prime}\right]$, with the property that $K\left[G^{\prime \prime}\right]$ contains the points in $G^{\prime \prime}$ which correspond to $G^{\prime}$ in $G$; (3) any pair of neighbors in $G^{\prime \prime}$ such that both are not in $K\left[G^{\prime \prime}\right]$, is mapped into a pair of neighbors in $G$. If $G_{1}$ and $G_{2}$ are any two graphs in the set associated with $G$ and $G^{\prime}$, we shall say $G_{1} \geq G_{2}$ whenever the points in $G$ corresponding to $K\left[G_{2}\right]$ are a subset of the points corresponding to $K\left[G_{1}\right]$, and $G_{1}>G_{2}$ whenever $G_{1} \geq G_{2}$ but $G_{1} \neq G_{2}$.

Theorem 4.1. Given $G$ and a subgraph $G^{\prime}$, the set of graphs associated with $G$ and $G^{\prime}$, if nonempty, form a lattice under the relation $\geq$.

The proof will be left to the reader. We shall denote by $h\left(G^{\prime \prime} ; G, G^{\prime}\right)$ the Moebius function evaluated for any $G^{\prime \prime}$ in the lattice of graphs associated with $G$ and $G^{\prime}$, while $f_{1}\left(\phi ; G^{\prime \prime}\right)$ will denote the Moebius function evaluated for $\phi$ on the lattice of subgraphs 1-dense in $G^{\prime \prime}$. The function $h_{1}$ whose domain is the set of graphs associated with $G$ and $G^{\prime}$ is defined by $h_{1}\left(G^{\prime \prime} ; G, G^{\prime}\right) \equiv(-)^{\sigma\left(K\left[G^{\prime \prime}\right]\right)+n}$, where $G^{\prime \prime}$ is any graph of the set.

Theorem 4.2. On the lattice formed by the set of graphs associated with $G$ and $G^{\prime}, h_{1}=h$.

If $G_{m}$ is the greatest element of the lattice $K\left[G_{m}\right]$ $=G_{m}$ and $\sigma\left(K\left[G_{m}\right]\right)=n$, so that $h_{1}\left(G_{m} ; G, G^{\prime}\right)=1$. If $G^{\prime \prime}<G_{m}$ and $w \equiv \sigma\left(K\left[G_{m}\right]\right)-\sigma\left(K\left[G^{\prime \prime}\right]\right)$ there are $\left(\begin{array}{c}w \\ k\end{array}\right)$ graphs $H$ which satisfy the condition that $G^{\prime \prime}<H$ $\leq G_{m}$ and $\sigma(K[H])-\sigma\left(H\left[G^{\prime \prime}\right]\right)=k$. Therefore

$$
\begin{aligned}
-\sum_{H>G^{\prime \prime}} & h_{1}\left(H ; G, G^{\prime}\right)=(-)^{\sigma\left(K\left[G^{\prime \prime}\right]\right)+n+1} \\
& \times \sum_{k=1}^{w}\left(\begin{array}{c}
w \\
k
\end{array}\right)(-)^{k}=(-)^{\sigma\left(K\left[G^{\prime \prime}\right]\right)+n}=h_{1}\left(G^{\prime \prime} ; G, G^{\prime}\right) .
\end{aligned}
$$

Since $h_{1}$ satisfies the recurrence relations which uniquely determine $h$, the theorem follows.

Let $R\left[G^{\prime \prime}\right]$ be the set of subgraphs 1-dense in $G^{\prime \prime}$ and $Q\left[G^{\prime \prime}\right]$ the set of all subgraphs in $R\left[G^{\prime \prime}\right]$ which contain at least one point from $K\left[G^{\prime \prime}\right]$.

Lemma 4.3. If $G^{\prime \prime}$ is any graph in the set associated with $G$ and $G^{\prime}$ such that $K\left[G^{\prime \prime}\right] \neq G^{\prime \prime}$, then

by 2.5 ,

$$
f_{1}\left(\phi ; G^{\prime \prime}\right)=(-)^{\sigma\left(G^{\prime \prime}\right)+1} \sum_{H \in R\left[G^{\prime \prime}\right]-Q\left[G^{\prime \prime}\right]}(-)^{\sigma(H)} .
$$

$$
\begin{aligned}
f_{1}\left(\phi ; G^{\prime \prime}\right)=(-)^{\sigma\left(G^{\prime \prime}\right)+1}\left[\sum_{H \in Q\left[G^{\prime \prime}\right]}(-)^{\sigma(H)}\right. & \\
& \left.+\sum_{H \in R\left[G^{\prime \prime}\right]-Q\left[G^{\prime \prime}\right]}(-)^{\sigma(H)}\right] .
\end{aligned}
$$

By 2.7, the first sum in the bracket can be expressed as a sum over families of subsets of $K\left[G^{\prime \prime}\right]$. If $C$ is such a family and $B(C)$ the union of the graphs in the family, $B(C)$ is a connected proper subgraph because $K\left[G^{\prime \prime}\right]$ is completely connected ${ }_{-}^{\ddagger}$ and $\neq G^{\prime \prime}$, and thus

$$
\sum_{B(C) \supset H \in R\left[G^{\prime \prime}\right]}(-)^{\sigma(H)}=0
$$

by 2.4. Since this is true for every family $C$ of subgraphs of $K\left[G^{\prime \prime}\right]$, we have

$$
\sum_{H \in Q\left[G^{\prime \prime}\right]}(-; \sigma(H)=0
$$

THeORem 4.4. If $G^{\prime \prime}$ is a graph belonging to the set associated with $G$ and $G^{\prime}$, then

1) If $G^{\prime \prime}-K\left[G^{\prime \prime}\right]$ is 1-dense in $G^{\prime \prime}$,

$$
\sum_{H \geq G^{\prime \prime}} h\left(H ; G, G^{\prime}\right) f_{1}(\phi ; H)=0 .
$$

2) If $G^{\prime \prime}-K\left[G^{\prime \prime}\right]$ is not 1-dense in $G^{\prime \prime}$,

$$
\sum_{H \geq G^{\prime \prime}} h\left(H ; G, G^{\prime}\right) f_{1}(\phi ; H)=(-)^{\sigma\left(G^{\prime \prime}\right)} .
$$

By 4.2 and 4.3 , we have for $G^{\prime \prime} \neq K\left[G^{\prime \prime}\right]$

$$
\begin{array}{r}
-\sum_{H>G^{\prime \prime}} h\left(H ; G, G^{\prime \prime}\right) f_{1}(\phi, H)=\sum_{\substack{H>G^{\prime \prime} \\
\left(-H^{\prime} \epsilon R[\mathrm{H}]-Q[\mathrm{H}]\right.}} \sum^{\sigma(K[H])+\sigma\left(H^{\prime}\right)}+(-)^{n+1} .
\end{array}
$$

If $H^{\prime}$ is any subgraph of $G^{\prime \prime}$ which is one of the set $S$ associated with $G$ and $G^{\prime}, H^{\prime}$ corresponds to a unique subgraph $H_{1}^{\prime} \subset G$. In turn $H_{1}^{\prime}$ corresponds to a unique subgraph $H^{\prime \prime}$ in any other graph of $S$, and these two correspondences determine a correspondence between $H^{\prime}$ and $H^{\prime \prime}$. If $H>G^{\prime \prime}$, every subgraph of $H-K[H]$ corresponds to a subgraph in $G^{\prime \prime}-K\left[G^{\prime \prime}\right]$. If $G^{\prime \prime}-K\left[G^{\prime \prime}\right]$ is not 1-dense, it contains no 1-dense subgraph, and so there can be no corresponding 1-dense subgraph in $H-K[H]$ and $R[H]-Q[H]$ is empty for $H \geq G^{\prime \prime}$. Therefore, if $G^{\prime \prime} \neq K\left[G^{\prime \prime}\right],-h\left(G^{\prime \prime} ; G, G^{\prime}\right) f_{1}\left(\phi ; G^{\prime \prime}\right)$ can be added to both members of (B), and then the double sum vanishes, proving part 2 for this case. If $G^{\prime \prime}=K\left[G^{\prime \prime}\right], f_{1}\left(\phi ; G^{\prime \prime}\right)=(-)^{n}$ and part 2 is obvious.

Suppose $G^{\prime \prime}-K\left[G^{\prime \prime}\right]$ is 1 -dense, and $H^{\prime} \in R\left[G^{\prime \prime}\right]-$ $Q\left[G^{\prime \prime}\right]$. If $w \equiv \sigma\left(G^{\prime \prime}-H^{\prime}-K\left[G^{\prime \prime}\right]\right)$ and $k=1, \ldots$ $w$, there are $\left(\begin{array}{c}w \\ b\end{array}\right)$ graphs $H>G^{\prime \prime}$ with the property that $K[H]=K\left[G^{\prime \prime}\right]+k$ and $H-K[H]$ contains a subgraph which corresponds to $H^{\prime}$. The contribution to the double sum in (B) of all the graphs which correspond to a particular $H^{\prime} \epsilon R\left[G^{\prime \prime}\right]-Q\left[G^{\prime \prime}\right]$ is

$$
\sum_{k=1}^{w}\left(\begin{array}{l}
w \\
k
\end{array}\right)(-)^{\sigma\left(K\left[G^{\prime \prime}\right)+k+\sigma\left(H^{\prime}\right)\right.}=(-)^{\sigma\left(K\left[G^{\prime \prime}\right]\right)+1+\sigma\left(H^{\prime}\right)} .
$$

(B) contains a similar contribution for every $H^{\prime} \epsilon R\left[G^{\prime \prime}\right]-Q\left[G^{\prime \prime}\right]$ except $G^{\prime \prime}-K\left[G^{\prime \prime}\right]$. Thus

$$
\begin{aligned}
& -\sum_{H>G^{\prime \prime}} h\left(H ; G, G^{\prime}\right) f_{1}(\phi ; H)=h\left(G^{\prime \prime} ; G, G^{\prime}\right) f_{1}\left(\phi ; G^{\prime \prime}\right) \\
& \left.-(-)^{\sigma\left(K\left[G^{\prime \prime}\right]\right)+1+\sigma\left(G^{\prime \prime}-K\left[G^{\prime \prime}\right]\right.}\right)+(-)^{n+1} .
\end{aligned}
$$

Since $\sigma\left(G^{\prime \prime}\right)=\sigma(G)=n$, part 1 follows.

\section{References}

[1] R. E. Nettleton, Some generalized theorems on connectivity (to be published).

[2] G. Birkhoff, Lattice theory, Am. Math. Soc. Colloq. Pub. 25 (1948).

[3] R. E. Nettleton, K. Goldberg, and M. S. Green, Dense subgraphs and connectivity, Can. J. Math 11, 262 (1959).

[4] P. Hall, Quar. J. 7, 134 (1936).

Washington, D.C.

(Paper 64B1-19) 